\title{
THE SCATTERING OF LIGHT FROM LIQUID HELIUM
}

\author{
W. F. VINEN and J. M. VAUGHAN \\ Department of Physics, University of Birmingham, England
}

\begin{abstract}
A review is given of the scattering of light by superfluid helium. A brief discussion is given first of the theory of Brillouin and Rayleigh scattering from an ordinary simple pure liquid. It is shown how this discussion must be modified in the case of the superfluid phase of pure ${ }^{4} \mathrm{He}$, and experimental observations of Brillouin scattering from first sound in liquid helium are described. The discussion is extended to the case of a superfluid mixture of ${ }^{3} \mathrm{He}$ and ${ }^{4} \mathrm{He}$, and a recent observation of Brillouin scattering from second sound in such a mixture is described. Recent work on Raman scattering from superfluid helium (two roton production) is then discussed, and in a final section a brief review is given of the special situation that obtains in the neighbourhood of the $\lambda$-point.
\end{abstract}

1. Introduction. - A liquid scatters light because of fluctuations (often thermal) in its refractive index, and a study of the intensity and spectrum of the scattered light yields information about the intensity and spectrum of these fluctuations. Fluctuations in a superfluid are of particular interest, and in this paper we aim to review the information that has been, and could be, obtained from a study of light scattering from the superfluid phase of liquid helium.

Experiments on the scattering of light from a liquid are usually difficult, for two reasons. First, the amount of light scattered is generally very small ; and secondly, a full examination of the spectrum of the scattered light requires, as we shall see, an instrument of very high resolving power. Thus extensive experiments have become possible only recently, following the development of the laser and following recent advances in high resolution spectroscopy and the techniques of light detection. In the case of liquid helium the amount of scattered light is extremely small (owing to the low temperature, and the small polarizability of the medium), with the result that experiments on the spectrum of light scattered by helium have begun to be carried out only within the past two or three years.

We begin our review with a brief discussion of the theory of Brillouin and Rayleigh scattering from an ordinary simple pure liquid ( $\S 2$ ). In $\S 3$ we show how this discussion must be modified in the case of a pure superfluid, and we describe the recent experimental observation of Brillouin scattering from first sound in liquid ${ }^{4} \mathrm{He}$. In $\S 4$ we extend the discussion to scattering from a superfluid mixture of ${ }^{3} \mathrm{He}$ and ${ }^{4} \mathrm{He}$, and describe a recent observation of Brillouin scattering from second sound in such a mixture. A form of second-order Raman scattering is possible in superfluid helium, involving the production of two rotons, and this effect will be discussed in $\S 5$. In $\S 6$, we discuss briefly the special situation that obtains in the immediate neighbourhood of the $\lambda$-point.

The bulk of our discussion will be concerned with scattering from helium that is close to thermal equilibrium. It is, however, possible to scatter light from helium in which extra fluctuations have been deliberately introduced, as occurs, for example, in stimulated Brillouin scattering. We shall consider situations of this kind at appropriate points in $\S \S 2-3$.

2. Light scattering from an ordinary simple liquid. - Suppose light of frequency $\omega_{0}$ and wave number $\mathbf{k}_{0}$ is incident on a simple pure liquid which can be characterized locally by a single refractive index $n(\mathbf{R}, t)=n_{\mathrm{av}}+\delta n(\mathbf{R}, t)$. Then, as is easily shown [1] by solving the electromagnetic wave equation, the intensity at distance $R$ of the scattered light, of frequency $\omega^{\prime}$ and wave number $\mathbf{k}^{\prime}$, is proportional to the quantity $S\left(\mathbf{Q}, \omega^{\prime}\right)$, where

$$
S\left(\mathbf{Q}, \omega^{\prime}\right)=\text { const. } \omega_{0}^{4} \frac{\sin ^{2} \varphi}{R^{2}}(\delta n)_{\mathbf{Q}, \Delta \omega}^{2} .
$$

$\mathbf{Q}$ is the change of wave vector, $\mathbf{k}^{\prime}-\mathbf{k}_{0}, \Delta \omega$ is the change of frequency, $\omega^{\prime}-\omega_{0}$, and $\varphi$ is the angle between the electric field in the incident light and the direction of $\mathbf{k}^{\prime} .(\delta n)_{\mathbf{Q}, \Delta \omega}^{2}$ is the Fourier transform in space and time of the correlation function

$$
<\delta n\left(\mathbf{R}+\mathbf{R}_{1}, t+t_{1}\right) \delta n(R, t)>,
$$

which is a function of $\mathbf{R}_{1}$ and $\mathbf{t}_{1}$ only. Thus any light scattered with wave number change $\mathbf{Q}$ and frequency change $\Delta \omega$ must be due to the presence within the liquid of a periodic modulation in the refractive index with wave number $\mathbf{Q}$ and frequency $\Delta \omega$. This result 
is easily understood physically. If there is no modulation in the refractive index there is no scattering, since the scattered wavelets from each element of the liquid combine destructively except in the forward direction. A modulation in the refractive index does, however, cause scattering because it acts as a diffraction grating, and the change in frequency is due simply to the Doppler effect associated with the moving pattern of the modulation. A study of the intensity of light scattered at $(\mathbf{Q}, \Delta \omega)$ is thus a measure of the intensity of the fluctuation of the refractive index of wave number $\mathbf{Q}$ and frequency $\Delta \omega$. For a simple pure liquid this refractive index is just proportional to the density, so the scattered light contains information about density fluctuations or, more precisely, about the density-density correlation function.

We note that with visible light the minimum value of the wavelength $2 \pi / Q$ is of order $2000 \AA$ and is large compared with the interatomic spacing in the liquid. The simplest type of fluctuation that we can consider is then one which preserves local thermodynamic equilibrium. The intensity of such a fluctuation is easily calculated [2]. For a density fluctuation of wave number $\mathbf{Q}$ the total intensity, irrespective of frequency, is given by simple statistical mechanics as (for unit volume of liquid)

$$
(\overline{\delta \rho})_{Q}^{2}=k_{B} T \rho\left(\frac{\partial \rho}{\partial p}\right)_{T}
$$

and so is proportional simply to the isothermal compressibility (we have assumed that the fluctuations can be described classically). The total scattered light intensity in the direction defined by the vector $\mathbf{Q}$ is then proportional to

$$
\begin{aligned}
S(\mathbf{Q})= & \int S\left(Q, \omega^{\prime}\right) \mathrm{d} \omega^{\prime}= \\
& =\text { const. } \omega_{0}^{4} \frac{\sin ^{2} \varphi}{R^{2}}\left(\frac{\mathrm{d} n}{\mathrm{~d} \rho}\right)^{2} k_{B} T \rho\left(\frac{\partial \rho}{\partial p}\right)_{T} .
\end{aligned}
$$

To obtain the spectrum of this scattered light we have to consider how the density fluctuation evolves in time. We recognise that a fluctuation in density of wave number $\mathbf{Q}$ can be decomposed into two «normal modes» : a wave which is essentially a pressure wave, at approximately constant entropy; and a wave which is essentially an entropy, or temperature, wave, at constant pressure (remember the thermodynamic identity : $\left.\mathrm{d} \rho=(\partial \rho / \partial p)_{S} \mathrm{~d} p+(\partial \rho / \partial s)_{\mathrm{p}} \mathrm{d} S\right)$. The first is just a sound wave, propagating with speed $u$. The light scattered by such a wave is Doppler-shifted, by an amount which is easily seen to be

$$
\pm 2 n[u / c] \omega_{0} \sin \theta / 2
$$

where $\theta$ is the angle of scattering. The second, which involves a density change through the coefficient of expansion, does not propagate, and, in the absence of dissipation, the light that is scattered by it suffers no shift in frequency. Thus the spectrum of the scattered light contains three lines : an undisplaced Rayleigh line, due to entropy fluctuations, and a Brillouin doublet due to thermally excited sound waves. When dissipation is taken into account, each of these lines is found to have a finite width : the Brillouin line width is determined by the attenuation of sound at the appropriate frequency; the Rayleigh line width is determined by the rate of decay of the entropy fluctuations by thermal conduction, i. e. by the thermal diffusivity of the medium. The relative intensities of the Rayleigh and Brillouin components must clearly depend on the coefficient of expansion of the liquid ; it may in fact be shown quite easily that [3]

$$
\frac{I_{R}}{I_{B}}=(\gamma-1)
$$

where $\gamma=C_{p} / C_{v}(\gamma-1$ is proportional to the square of the coefficient of expansion). We note that the intensity of the Brillouin components is proportional to the adiabatic compressibility of the liquid.

A quantitative formulation of this scattering theory is most elegantly expressed in terms of generalized susceptibilities and the fluctuation-dissipation theorem [2]. To calculate the density-density correlation function (from which (2) is derived), we imagine first that the liquid is acted upon by a suitable force which generates a density fluctuation of wave number $\mathbf{Q}$ (the force $F$ should be such that the change in energy is of the form $F \mathrm{~d} \rho$ ). We thus calculate the susceptibility $\chi=\delta \rho / F$. The density-density correlation function is then given by the fluctuation-dissipation theorem, which in the classical limit takes the form

$$
(\delta \rho)_{\mathbf{Q}, \Delta \omega}^{2}=\frac{2}{\pi} k_{B} T \frac{\chi^{\prime \prime}(Q, \Delta \omega)}{\Delta \omega}
$$

where $\chi^{\prime \prime}$ is the imaginary part of $\chi$. In the case where there is approximate local thermodynamic equilibrium, $\chi$ can be calculated from the hydrodynamic equations for the fluid [4].

In their broad features the predictions of this theory are in agreement with experiment [5]. There are, however, discrepancies in detail; for example, the spectrum of the light scattered from some liquids contains in addition to the three lines we have described a background centred on zero frequency shift but covering quite a wide frequency range [6]. To understand why such discrepancies exist we must recognize that our assumption of local thermodynamic equilibrium within a fluctuation may be incorrect $\left({ }^{1}\right)$. It is perfectly possible to have fluctuations which are not described in such simple terms. For example, we may have molecules in our liquid which can possess both kinetic energy of translation and energy of internal vibration, and we can imagine a

(1) We do not consider complications which arise if the molecules of the liquid have an anisotropic polarizability. 
fluctuation in which the relative amounts of these two contributions to the total energy of the system do not correspond to local equilibrium. Such a fluctuation may not propagate, in which case it would give rise to scattered light with frequency centred on the incident frequency and a line width of order the reciprocal of the relaxation time involved in the fluctuation [7]. If this time is short, the effect will be seen as a background scattering covering a wide frequency range. The kind of effect that we are describing here is one that gives rise to a coefficient of second viscosity, and one which causes therefore attenuation and dispersion of sound. The effects on light can, in fact, be taken into account by including in the hydrodynamical equations used to obtain the susceptibility $\chi$ a coefficient of second viscosity with the correct frequency dependence [8].

We see therefore that the spectrum of the light scattered by a liquid contains useful information about the liquid. Observations on the Brillouin doublet yield values of the velocity and attenuation of sound at the appropriate frequency, which is typically of order $2-4 \mathrm{GHz}$ ( $90^{\circ}$ scattering), and which is therefore hard to study by more conventional techniques. Other features of the spectrum yield information about the thermal diffusivity of the medium and about internal relaxation processes.

In our discussion so far of light scattering from a simple liquid we have assumed that the liquid is not disturbed significantly from equilibrium, and that the scattering fluctuations are therefore those associated with the equilibrium state of the liquid (thermal scattering). Two types of experiment have been carried out in which this condition has been violated, and we mention them briefly.

In the first, sound waves are deliberately introduced into the liquid by means of a transducer, and light is scattered from these sound waves. The intensity of the sound wave at a particular wave number can then be much larger than in thermal equilibrium, and the intensity of the light scattered is correspondingly larger. In the second type of experiment, the incident light is of very high intensity, so that non-linear effects become significant. One such non-linear effect, which is of interest here, is stimulated Brillouin scattering. This effect may be understood in classical terms as follows. The electric field in the incident light combines with that in the Brillouin scattered light to produce a « beating » wave in the electric field with frequency $\Delta \omega$ and wave number $\mathbf{Q}$. This latter electric field exerts a periodic electrostrictive force on the liquid, proportional to the square of the field; if the amplitude of the light is large enough this process will lead to the coherent generation of sound at frequency $\Delta \omega$ and wave number $\mathbf{Q}$ and to greatly increased scattering. The effect sets in when the incident light power exceeds a threshold value, and the effect can be used to generate hypersonic waves of very high amplitude.
3. Light scattering from a pure superfluid. - We now examine how the discussion of $\S 2$ must be modified in the case of the superfluid phase of pure ${ }^{4} \mathrm{He}$. We assume first that the fluctuations can be taken to involve situations of local thermodynamic equilibrium, and that the fluid as a whole is not disturbed significantly from equilibrium.

The result (4) for the total scattered intensity still holds, but the spectrum of the scattered light is modified [2]. This modification arises because a fluctuation in the entropy, at constant pressure, is no longer nonpropagating; such a fluctuation is just what we find in a second sound wave, and the fluctuation will propagate with the speed, $u_{2}$, of second sound. Thus in the spectrum of the scattered light the undisplaced Rayleigh line disappears, and is replaced by a doublet, with frequency shift $\pm 2 n\left(u_{2} / c\right) \omega_{0} \sin \theta / 2$. The Brillouin doublet, due to first sound, is unchanged. The doublets have line widths that are determined by the attenuation of the relevant sound at the frequency concerned.

The ratio of the scattered intensity due to entropy fluctuations (second sound) to that due to pressure fluctuations is still given approximately by (5). As we shall see, the latter intensity is only observable with difficulty, and except very near the $\lambda$-point (which we discuss in $\S 6)$ the value of $(\gamma-1)$ for superfluid helium is much less than unity. Thus, unfortunately, the scattering due to second sound is unobservably small, at least with present techniques. The physical reason is clear : in a second sound wave the total density of the liquid changes by only a very small amount, and the wave is therefore only very weakly coupled to an electromagnetic wave $\left({ }^{2}\right)$.

Quantitative measurements of the total light scattered by superfluid helium were first made by Lawson and Meyer [11], using a high-pressure mercury arc lamp as a source of light and a cooled photomultiplier as a detector. The amount of light scattered is very small, so that the experimental error was as high as $20 \%$, but within this error formula (4) for the total scattering was verified for temperatures in the range $1.5 \circ \mathrm{K}$ to $4.2 \mathrm{~K}$. More recently this experiment has been repeated by Grimm and Dransfeld [12], with a He-Ne laser as a source. They were able to verify formula (4) below the $\lambda$-point with a relative accuracy of $2 \%$ (absolute accuracy $15 \%$ ); above the $\lambda$-point there was some additional, presumably spurious, scattering, due perhaps to boiling.

Two experimental observations of the spectrum of light scattered from pure superfluid ${ }^{4} \mathrm{He}$ have been made recently, one by Greytak, St. Peters and Benedek [13] and one by Pike et al. [14]. We shall describe briefly the second of these observations.

We note first that for scattering at $90^{\circ}$ the expected

(2) It has been suggested, however, by Bertolotti et al. [10] that the effect of second sound might be just detectable in the photon statistics of the scattered light. 
frequency shift associated with the first sound Brillouin doublet is only about $700 \mathrm{MHz}$, so that a spectrometer of high resolving power, capable of operating with very low light intensities, is required. In the work of Pike et al. measurements were made with a plane Fabry-Perot etalon of $50 \mathrm{~mm}$ diameter and $662 \mathrm{~mm}$ spacing. Light from the liquid helium that passed into a region at the centre of the Fabry-Perot fringe system was detected by photoelectron counting with a cooled photomultiplier of dark count 0.45 electrons per second. The spectrum was scanned by imposing a small oscillation at $6 \mathrm{~Hz}$ on one etalon plate, and the counts from the photomultiplier were stored digitally in a multichannel scaler. The light source was an argon-ion laser with a continuous output of about $300 \mathrm{~mW}$. The total count rate due to the scattered light was a few electrons per second. An acceptable spectrum could be obtained after about 30 minutes recording.

The measurements show clearly the existence of a Brillouin doublet due to first sound, and the observed splitting agrees within an experimental error of about $0.5 \%$ with that deduced from the known velocity of sound. As expected there is no observable scattering either at the incident frequency or due to second sound. At most temperatures in the range studied (above $1.25 \mathrm{~K}$ ), the observed line width was instrumental, but at the lower temperature there was observable additional broadening consistent with the attenuation of sound measured by other methods. It should be added that in the comprehensive investigation of Greytak et al. [13] instrumental broadening was much reduced and attenuations were measured with an accuracy of $\pm 10 \%$ over a wide temperature range. Additionally, the line shifts were measured with high precision $( \pm 0.1 \%$ ), although it did not prove possible to eliminate a good deal of spurious scattering at the incident frequency.

In our discussion so far of light scattering from superfluid helium we have confined our attention to fluctuations that preserve local thermodynamic equilibrium. As in the case of an ordinary liquid, however, there may also be fluctuations that involve transfer of energy between the different modes of internal motion of the liquid at any particular point in space. We know indeed that such a situation can arise in helium since the division of the thermal energy between the phonons and rotons can be out of equilibrium. This possibility leads to the existence of a large coefficient of second viscosity in helium and hence to attenuation and dispersion of sound. In the present context it leads to the possible existence of non-propagating modes of fluctuation and hence to a line in the scattered spectrum that is centred on the incident frequency and has a width of the order of the reciprocal of the dominant relaxation time involved in the phonon-roton interactions. To our knowledge no calculations on this effect have yet been carried out, and it has not been observed experimentally, but it would be interesting to pursue the idea further.

We now mention briefly some experiments that have been carried out on superfluid helium which is not in thermal equilibrium. Van den Berg et al. [15] observed the diffraction pattern of light scattered from an injected $1 \mathrm{MHz}$ sound wave. From the known frequency of the injected wave, and the wave vector determined from the diffraction pattern, the sound velocity could be calculated. Scattering of light from first sound waves generated by a transducer at 556 and $723 \mathrm{MHz}$ has been observed by Woolf et al. [16]. The light source was a laser, but observation of the scattered light was made by mixing this light with some of the incident light and allowing the mixture to fall on a square-law photodetector. The mixing gives rise to beating at the difference frequency (equal to the frequency of the sound wave), and this in turn gives rise to a detectable microwave output from the photodetector. Measurement of the scattering angle for given frequency gave a value for the velocity of first sound, and measurement of what is, in effect, the line width gave the attenuation. Stimulated (first sound) Brillouin scattering in liquid helium has been observed by Winterling et al. [17] and by Abrikosova and Bochkova [18]; the threshold power has been measured, and agrees with theory except for an anomalous increase just below $1.3 \mathrm{oK}$ (the theoretical value involves apparently only quantities like the magnitude of the electrostriction that are of no interest in connection with the superfluid properties). The attenuation of phonons generated by stimulated Brillouin scattering has been measured in an ingenious experiment by Heinicke et al. [19]. In this experiment the light from two successive pulses from a $Q$-switched ruby laser is focussed onto a small region in the helium. The first pulse is of high power and generates phonons of frequency $650 \mathrm{MHz}$. The second pulse follows after a time interval of $42 \mathrm{~ns}$ and is much smaller intensity (less than the threshold for stimulated Brillouin scattering). This second pulse is scattered by the phonons remaining from the action of the first pulse; a measurement of the strength of this scattering will therefore give information about the relative number of phonons left and hence about their attenuation.

These new methods for measuring the attenuation and velocity of sound at high frequencies $(\sim 700 \mathrm{MHz})$ are potentially attractive, since conventional microwave ultrasonic methods are difficult, especially if attenuations are high. Unfortunately the experimental attenuations obtained both by Woolf et al. [16] and by Heinicke et al. [19] are in poor quantitative agreement with those obtained by Imai and Rudnick [20] by more conventional microwave ultrasonic methods at $1 \mathrm{GHz}$, at least at low temperatures. The reason is not clear : it is tempting to suppose that the very high ultrasonic amplitudes involved in these optical experiments might be troublesome, but the experimental results were shown to be independent of this ampli- 
tude. It is interesting that within the experimental accuracy the attenuation measurements from thermal Brillouin scattering [13] are in better agreement with those of Imai and Rudnick.

We have noted that in pure superfluid ${ }^{4} \mathrm{He}$ thermal Brillouin scattering from second sound should be too small to be observable with present techniques. However, scattering from second sound of large amplitude injected with a transelucer has been observed by Jacucci and Signorelli [21], using a heterodyne beat technique similar to that of Woolf et al. [16] already described. Full details of this work have not been published. A theory of stimulated Brillouin scattering from second sound has been given by Tsintsadze [22], but the effect has not yet been observed.

4. Light scattering from a superfiuid ${ }^{3} \mathrm{He}-{ }^{4} \mathrm{He}$ mixture. - It is known that it is possible to dissolve considerable quantities of ${ }^{3} \mathrm{He}$ in superfluid ${ }^{4} \mathrm{He}$, the mixture remaining superfluid, and, as we shall see, it is of interest to study the scattering of light by such a mixture.

We recall that the ${ }^{3} \mathrm{He}$ atoms do not participate in superflow [23], and that with the thermal excitations they form part of the normal fluid. In a second sound wave they move with the normal fluid, and therefore in such a wave there must be significant fluctuations in the ${ }^{3} \mathrm{He}$ concentration. Fluctuations in the concentration will be accompanied by fluctuations in the refractive index, and it seems likely therefore that second sound in a mixture will scatter light much more strongly than in pure ${ }^{4} \mathrm{He}$. It seems likely, indeed, that thermal scattering from second sound might be observable in a mixture without too much difficulty.

This expectation is confirmed by detailed calculations. In a mixture the refractive index depends significantly on both the mass density $\rho$ and the mass concentration $c$. It follows that a mean square fluctuation in the refractive index is given by

$$
\begin{aligned}
& \overline{(\delta n}^{2}=\left(\frac{\partial n}{\partial c}\right)_{\rho}^{2} \overline{(\delta c)^{2}}+2\left(\frac{\partial n}{\partial c}\right)_{\rho}\left(\frac{\partial n}{\partial \rho}\right)_{c} \overline{\delta c \overline{\delta \rho}}+ \\
& +\left(\frac{\partial n}{\partial \rho}\right)_{c}^{2}{\overline{(\delta \rho)^{2}}}^{2}
\end{aligned}
$$

so that it is necessary to calculate three correlation functions, of the forms $\langle\delta \rho \delta \rho\rangle,\langle\delta \rho \delta c\rangle$, $\langle\delta c \delta c\rangle$. This can be done from the hydrodynamic equations for the mixture [24], using, for example, the Kadanoff-Martin method [4]. Actual calculations have been carried out by Gorkov and Pitaevskii [25], by Ganguly and Griffin [26], and by the present authors [27].

It is clear from these calculations that there are three types of fluctuation that contribute to the light scattering: one which corresponds to first sound; one which corresponds to second sound; and one which corresponds to a special kind of concentration fluctuation, taking place at constant pressure and constant ${ }^{4} \mathrm{He}$ chemical potential. The first two give rise of course to spectral doublets with frequency shifts $\pm 2 n\left(u_{1} / c\right) \omega_{0} \sin (\theta / 2), \pm 2 n\left(u_{2} / c\right) \omega_{0} \sin (\theta / 2)$ the third is non-propagating and gives rise therefore to an undisplaced line. Over a range of fairly large ${ }^{3} \mathrm{He}$ concentrations the five spectral lines are predicted to be of comparable intensity, although the three calculations mentioned yield values for the relative intensities that are not in detailed agreement with one another. The line widths are determined by the damping coefficients of the fluctuations, which in turn are determined by the formidable collection of transport coefficients that are relevant to ${ }^{3} \mathrm{He}_{-}^{4} \mathrm{He}$ superfluid mixtures [24].

Preliminary experiments on light scattering from ${ }^{3} \mathrm{He}-{ }^{4} \mathrm{He}$ mixtures have been reported by Pike et al. [28]. The method was the same as that described in $\S 3$ in connection with thermal scattering from pure ${ }^{4} \mathrm{He}$. A mixture containing 17 molar $\%{ }^{3} \mathrm{He}$ was examined at a temperature of about $1.25{ }^{\circ} \mathrm{K}$, and a typical spectrum is shown in figure 1 . The frequency scale

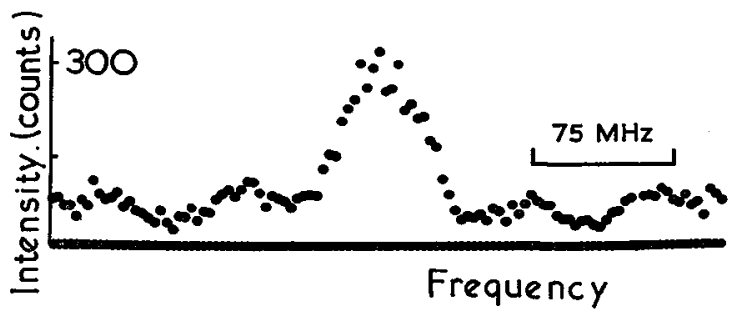

FIG. 1. - Brillouin scattering from a $17 \%$ (molar) mixture of ${ }^{3} \mathrm{He}$ in ${ }^{4} \mathrm{He}$ at $1.25 \circ \mathrm{K}$. The first-sound scattered light is shifted \pm 2.95 orders $( \pm 669 \pm 8 \mathrm{MHz})$ and overlaps at the centre. The second-sound scattering is five times weaker and shifted \pm 0.34 orders $( \pm 77 \pm 6 \mathrm{MHz}$ ). The outer peaks are secondsound scattering from adjacent orders.

in the figure covers about 1.5 orders of the FabryPerot etalon. The central peak is due largely to light scattered from first sound, shifted by \pm 2.95 orders $( \pm 669 \pm 8 \mathrm{MHz}$ ) and therefore overlapping any light of unshifted frequency. The inner smaller peaks are, we believe, due to second sound and are shifted \pm 0.34 orders $( \pm 77 \pm 6 \mathrm{MHz}$ ). These shifts correspond to speeds of first and second sound of $224 \pm 3 \mathrm{~ms}^{-1}$ and $26 \pm 2 \mathrm{~ms}^{-1}$, which are in good agreement with values obtained by conventional methods [29, 30] and which therefore confirm our interpretation of the spectrum. The outer smaller peaks are due to second sound in adjacent orders. Experiments with a different etalon spacing, to detect the unshifted line, have not yet been carried out. The observed relative intensities are consistent with the calculations of Gorkov and Pitaevskii [25] and of the authors [27] but not with those of Ganguly and Griffin [26] (the two former calculations yield results that are numerically rather similar). No measurements of line width have yet been made. 
We note that the observation of light scattering from a ${ }^{3} \mathrm{He}-{ }^{4} \mathrm{He}$ mixture provides a new method for measuring the velocity and attenuation of second sound. The method is potentially valuable because the frequency of the second sound is much higher (typically $70 \mathrm{MHz}$ ) than can be achieved with ordinary second sound transducers, and because much higher attenuations can be measured (see $\S 6$ ).

5. Raman scattering from superfluid ${ }^{4}$ He. - Brillouin scattering from superfluid ${ }^{4} \mathrm{He}$ can, of course, be regarded (roughly) as a process in which an incoming photon is scattered off the liquid with the creation or destruction of an excitation in the form of a phonon. Conservation of momentum requires that the phonon wave number be equal to the change in photon wave number; this change cannot exceed $4 \pi / \lambda$ (back scattering), where $\lambda$ is the wavelength of the light, so that the only excitations that can be studied are those with rather small wave number compared with the reciprocal of the interatomic spacing $\left({ }^{3}\right)$. The interesting roton part of the excitation spectrum is therefore completely inaccessible.

This limitation is not present in a process in which two excitations are involved. Suppose the scattering involves the creation of two excitations. The momentum condition is simply that the vector sum of the wave vectors of the two excitations be equal to the change in wave vector of the light. If the two excitations have wave numbers comparable with that of a typical roton, the condition is practically that the wave vectors of the two excitations be equal in magnitude and opposite in direction (see Fig. 2).

The probability that such a process will occur is given by the product of a basic interaction between the photon and the excitation and the joint density of states for the two excitations. For superfluid helium this density of states has three maxima, corresponding to the pair of excitations being in the three regions A, B and C of the excitation spectrum (Fig. 2). Thus, if we assume that the basic interaction does not vary violently with wave number, we expect that the spectrum of the scattered light due to two-excitation processes will be continuous, but with peaks at energy shifts equal to $2 \Delta_{\mathrm{A}}, 2 \Delta_{\mathrm{B}}$, and $2 \Delta_{\mathrm{C}}$ [31]. The processes involved here are analogous to those involved in the second-order Raman effect in the scattering of light from crystals.

Experimental observation of two of the predicted peaks has recently been reported by Greytak and Yan [32]. The helium was illuminated with a $1 \mathrm{~W}$ argon laser, and the spectrum examined with an Ebert-grating monochromator, using photoelectron counting with a cooled photomultiplier. The frequency

(3) This is equivalent to the statement that the diffraction of light by the density fluctuation associated with the excitation is possible only if the wavelength of the excitation is longer than half the wavelength of light.

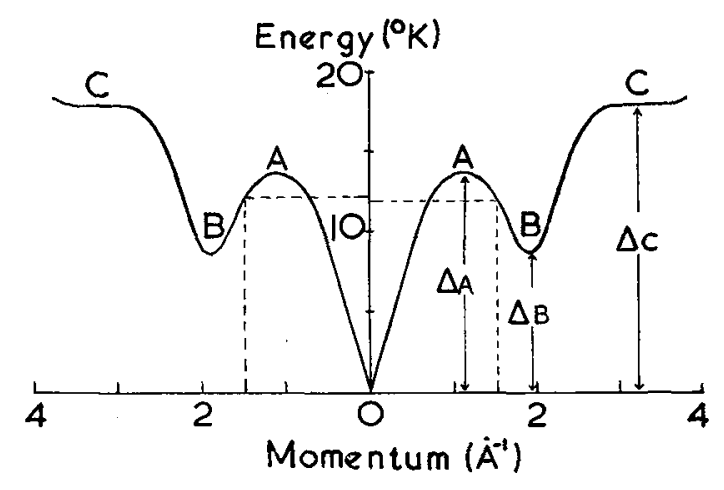

FIG. 2. - The dispersion curve for liquid $\mathrm{He}^{4}$ taken from neutron inelastic scattering data. Regions of high density of states are labelled A, B and C. The dotted lines show a possible pair of excitations, of nearly equal and opposite momenta, created in the liquid during the scattering of a photon.

shifts involved are now of order $400 \mathrm{GHz}\left(14 \mathrm{~cm}^{-1}\right)$, and therefore very high resolution is not required. The spectrum obtained at $90^{\circ}$ and $1.16^{\circ} \mathrm{K}$ is shown in figure 3. The strong peak at an energy shift of

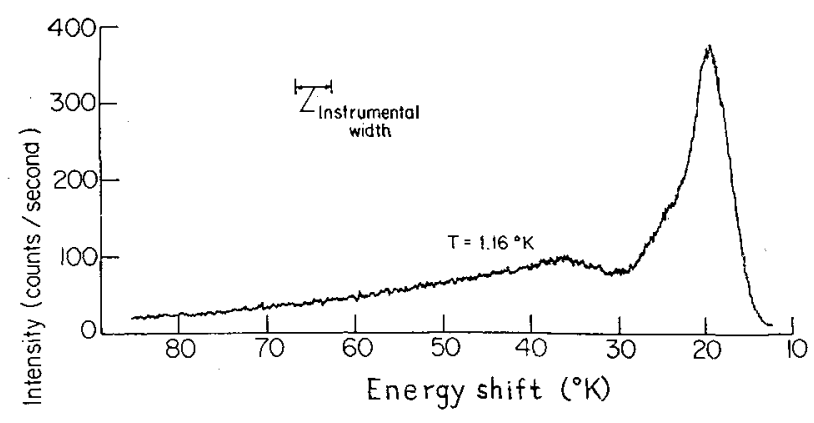

FIG. 3. - The Raman spectrum of liquid ${ }^{4} \mathrm{He}$ at a scattering angle of $90^{\circ}$, in a direction parallel to the electric field vector of the incident light (Greytak and Yan, reference 32).

$18.5^{\circ} \mathrm{K}$ corresponds to two-roton sreation at $2^{\prime} \Delta_{\mathrm{B}}$; the difference between $18.5^{\circ} \mathrm{K}$ and the accepted value of $2 \Delta$ for the roton minimum, $17.30 \mathrm{o}$, is due to an instrumental shift. There is also a less well-defined peak at $36 \mathrm{~K}$ which is probably to be identified with $2 \Delta_{\mathrm{C}}$. No clear peak is observed corresponding to $2 \Delta_{\mathrm{A}}$. At higher temperatures the peaks broaden, owing to shortened lifetime of the excitations, and the peak at $2 \Delta_{\mathrm{C}}$ soon disappears. The position of the rotonminimum peak $\left(2 \Delta_{B}\right)$ is observed to shift to lower energies with increasing temperature, which is consistent with the behaviour observed in neutron diffraction experiments [33].

A calculation of the intensity of Raman scattering by liquid helium has been made by Stephen [34]. He obtained results that are in good general agreement with experiment, both in the absolute magnitudes of the scattering and in the relative magnitudes for the three possible peaks (in particular the failure to observe any clear peak at $2 \Delta_{\mathrm{A}}$ is consistent with Stephen's results). According to Stephen's calculation 
the physical mechanism of the scattering is as follows. Suppose the scattering involves the creation of rotons of wave numbers $\mathbf{Q}_{1}$ and $\mathbf{Q}_{2}$. Each roton involves a density wave of the appropriate wave number. The electric field in the incident light (wave number $\mathbf{K}$ ) induces a polarization in the medium. This polarization is spatially modulated with wave number $\mathbf{Q}_{\mathbf{1}}$ (say), and so produces a spatially modulated electric field. We now imagine that this spatially modulated electric field acts back on the medium which we now regard as modulated in density by the second roton, wave number $\mathbf{Q}_{2}$. The resulting polarization has a component with wave number $\left(\mathbf{K}-\mathbf{Q}_{\mathbf{1}}-\mathbf{Q}_{2}\right)$ and frequency $\left\{c K-\omega\left(Q_{1}\right)-\omega\left(Q_{2}\right)\right\}$ (in an obvious notation), which produces the scattered wave. We see that the process involves two successive polarizations of the medium, and therefore the scattered intensity is proportional to the fourth power of the polarizability and not to the second power, as in Brillouin scattering. Another consequence of the existence of two stages in the process is that the scattered light is largely depolarized (unlike the Brillouin scattered light), and this depolarization was indeed observed by Greytak and Yan.

This optical method of investigating the energy spectrum of the excitations in superfluid helium will be a valuable addition to the older method based on neutron diffraction. We can see that the optical method gives less complete information, but it has two advantages : it has better precision thant the neutron method (the roton minimum energy, for example, can be determined more accurately); and it can be used in the presence of ${ }^{3} \mathrm{He}$ (which absorbs neutrons strongly). Measurements of the roton minimum energy as a function of ${ }^{3} \mathrm{He}$ concentration would be particularly interesting, because it has been suggested recently, on the basis of the analysis of some fourth sound measurements [35], that this energy may decrease quite rapidly with increasing ${ }^{3} \mathrm{He}$ concentration.

6. Scattering near the $\lambda$-point. - It is well known that light is very strongly scattered by a fluid in the immediate neighbourhood of the gas-liquid critical point (critical opalescence) [36]. The reason can be seen from equation (4) and from the fact that the isothermal compressibility of the fluid obviously tends to infinity at the critical point. In contrast to the situation obtaining under ordinary conditions, the scattering is strongly peaked in the forward direction. This is because the effective compressibility decreases with decreasing wavelength of the scattering fluctuation in density, the compressibility being infinite only in the limit of long wavelengths [37]. The spectrum is such that most of the scattered light is concentrated into the Rayleigh line, owing to a divergence in the value of $\gamma$ at the critical point. The width of the Rayleigh line is proportional to the thermal diffusivity of the medium, $K / C_{\mathrm{p}}$; the specific heat $C_{\mathrm{p}}$ diverges to infinity at the critical point, and diverges more rapidly than the conductivity $K$, so that the Rayleigh line becomes very narrow as well as very strong. The large fluctuations occurring in the region of the critical point of a fluid play an important role in determining the properties of the fluid in that region; a study of the scattering of light from a fluid near its critical point is therefore very useful since it gives direct information about the spectrum and intensity of these critical fluctuations. Similar considerations apply near the consolute point of a binary liquid mixture.

The $\lambda$-point of liquid helium is, of course, a type of critical point, and it is of interest therefore to ask whether a situation ought to occur here that is similar to that at the classical critical point. We shall see that it ought, although experimental observation of it is likely to prove difficult.

We remark first that experimental evidence [38] suggests that the isothermal compressibility of liquid helium (for long wavelengths) probably becomes infinite at the $\lambda$-point. Thus, as in the case of the gas-liquid critical point, the total scattered light is likely to increase considerably in the immediate neighbourhood of the $\lambda$-point ; and the scattered light is likely to be peaked in the forward direction, for reasons which are similar to those applying to the gas-liquid critical point. Unfortunately, however, the divergence in the compressibility is very weak: a rough calculation based on available experimental data [38] suggests that to obtain an increase in total scattering by a factor of only two would require at high pressures a temperature difference of only about $10^{-5} \mathrm{~K}$, and the situation is likely to be even worse at low pressures. It is presumably for this reason that no anomaly in the total scattering at the $\lambda$-point has yet been seen experimentally $[11,12]$. But it seems likely that with suitable experimental care this effect on the total scattering could be observed, and a study of it would give valuable confirmatory evidence about the behaviour of the isothermal compressibility.

We now turn to the expected spectrum of the scattered light, and consider first the effect on the first sound Brillouin doublet of moving towards the $\lambda$-point. Direct measurements of the velocity of first sound [39] show that at low frequencies $u_{1}$ passes through a minimum value of about $218 \mathrm{~ms}^{-1}$ just below the $\lambda$-point $\left(u_{1}=238 \mathrm{~ms}^{-1}\right.$ at $\left.T=0\right)$. Thus the adiabatic compressibility increases only a little near the $\lambda$-point, so that there will be only a slight increase in the intensity of the first sound Brillouin doublet. However, there is a large peak in the attenuation of sound [20] at or close to the $\lambda$-point, and this will cause considerable broadening of the Brillouin lines.

No detailed experimental observations have yet been made of the spectrum of light scattered thermally by liquid helium near the $\lambda$-point, although a qualitative observation of the increased line width in the first sound doublet was made by St Peters [13]. The only detailed optical observations that have been made in 
the neighbourhood of the $\lambda$-point are those of Heinicke et al. [19], which we described in $\S 3$, and in which the decay was observed of phonons injected by stimulated Brillouin scattering. Measurements of the decay rate, and hence of the attenuation, were made close to the $\lambda$-point, and they provide useful data with which to compare recent theories of transport processes close to the $\lambda$-point (see, e. g., references 40,41 ). We may remark again that light scattering provides a useful tool for studying the velocity and attenuation of first sound in helium at high frequencies, even in the presence of large attenuation, and further study of the $\lambda$-point by this technique seems worthwhile.

Since, as we see, the intensity of the first sound Brillouin doublet increases only slightly at the $\lambda$ point, the expected large increase in the total scattering must be associated with a large increase in the scattering in the second sound doublet. Formally, this is due to a divergence in the value of $\gamma$. Physically, the increase is associated with the vanishing of the velocity of second sound at the $\lambda$-point. The critical fluctuations at the $\lambda$-point are essentially second sound waves, and it is these fluctuations that cause the extra scattering.

We have already seen that at temperatures well below the $\lambda$-point the intensity of the second sound doublet is unobservably small. We see now, however, that this intensity should grow as we approach the $\lambda$-point and should become very large at a temperature sufficiently close to the $\lambda$-point. Unfortunately, as we have seen, the temperature does in fact have to be extremely close to the $\lambda$-point $\left(\sim 10^{-5} \circ \mathrm{K}\right.$ at high pressures) before the extra scattering becomes comparable with that from first sound, and, to our knowledge, this effect has not yet been observed.

It is of considerable interest to discuss the expected spectrum of the light scattered by the critical fluctuations in the neighbourhood of the $\lambda$-point, since this is directly related to the spectrum of the critical fluctuations themselves. In our discussion we shall use the results of recent theories based on the idea of dynamical scaling (see, particularly, reference 40 ).

It is important to realise at the outset that the well-known second sound spectrum

$$
\omega^{2}=u_{2}^{2} Q^{2} ; \quad u_{2}^{2}=\frac{T S^{2}}{C_{p}} \frac{\rho_{s}}{\rho_{n}}
$$

applies only in the so-called hydrodynamic regime; this means that it will apply in the neighbourhood of the $\lambda$-point, only if the wavelength $1 / Q$ is large compared with a temperature dependent correlation length $\xi(T)$, which, for helium, is given by

$$
\xi(T)=\xi_{0}\left|\frac{T_{\lambda}-T}{T_{\lambda}}\right|^{-2 / 3}
$$

where $\xi_{0}$ is a constant equal to $0.268 \AA$. Equation (8) implies that the space and time Fourier transform of the density-density correlation function contains a contribution of the form (in its dependence on $\omega$ )

$$
\frac{A \omega^{2}}{\left(\omega^{2}-Q^{2} u_{2}^{2}\right)^{2}+D \omega^{2}},
$$

where we have included a damping coefficient $D$, which increases as we approach the $\lambda$-point. In the opposite limit, $Q \xi(T) \gg 1$, we are in the critical region; the dispersion relation for the critical fluctuations is then completely different, and the correlation function is believed to take the form [40]

$$
\frac{A \omega^{2}}{\left[\omega^{2}-\frac{a Q^{3}}{\ln \left(\frac{1}{Q \xi_{0}}\right)}\right]^{2}+\frac{4 \omega^{2} a Q^{3}}{\ln \left(\frac{1}{Q \xi_{0}}\right)}}
$$

(we note that this implies a dispersion relation with critical damping; the constant $a$ is of order $\left.0.25 \mathrm{~cm}^{3} \mathrm{~s}^{-2}\right)$. As long as we are in the critical region, $Q \xi(T) \gg 1$, équation (11) is believed to apply both below and above the $\lambda$-point. In the hydrodynamic region, $Q \xi(T) \ll 1$, above the $\lambda$-point, the fluctuations are governed simply by thermal diffusion, and the correlation function takes the form

$$
-\frac{A \omega^{2}}{\omega^{4}+\omega^{2} Q^{4} K^{2} / C_{p}} .
$$

The functions (10), (11), (12) must change continuously from one to another as we move in and out of the critical region by varying the temperature for a given $Q$.

Let us now consider the scattering of light by the critical fluctuations at a given scattering angle; i. e. at a given $\mathbf{Q}$. The spectra in the three possible temperature regions (hydrodynamic, $T<T_{\lambda}$; critical ; hydrodynamic, $T>T_{\lambda}$ ) are given simply by (10), (11) and (12). In the low temperature hydrodynamic region (10) leads to a well-defined second sound doublet (Fig. 4, curve (a)), but with a damping (line width) that must increase towards critical as we approach the critical region. In the critical region, (11) holds, and we get the spectrum (b) of figure 4 . As we raise the temperature into the high temperature hydrodynamic region, the dip at $\omega=0$ must get narrower, and eventually the spectrum will tend to the simple Lorentzian (c) of equation (12), although some central dip will apparently remain.

We notice that the predicted spectrum shows no discontinuous change at the $\lambda$-point, except in the limit $Q=0$. This is an example of a basic idea that there is no discontinuous change in the properties of a superfluid at a finite wave number as the temperature is raised through the transition. Thus some « superfluid» properties must still exist at a finite wave number for $\bar{T}>T_{\lambda}$, and the predicted central dip in the spectrum of the scattered light is an example of such a property. 

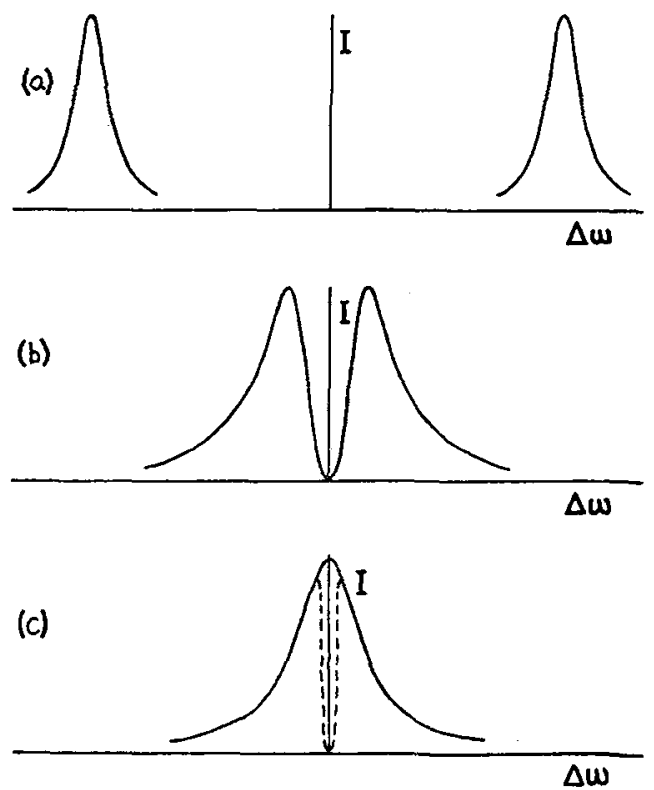

FIG. 4. - Sketches showing expected spectra of light scattered by entropy fluctuations near the $\lambda$-point ; intensity plotted against frequency shift. (a) $T<T_{\lambda}$; (b) $T=T_{\lambda}$; (c) $T>T_{\lambda}$. The curves are normalized to give the same maximum intensities. There may of course be other contributions to the total observed scattered intensities.
To see the predicted angular dependence of the scattering requires a more detailed analysis than is possible here, but it turns out that in the critical region there is a tendency for peaking in the forward direction, as in the case of the gas-liquid critical point.

These predicted features of the spectrum of light scattered near the $\lambda$-point have not yet been observed, and we must ask how difficult an observation would be. We note that the maximum value of $Q$ in the scattering of visible light is about $10^{5} \pi \mathrm{cm}^{-1}$. At this wave number one enters the critical region at a temperature difference $\left(T_{\lambda}-T\right)$ given by $\xi(T) Q=1$; i. e. at a temperature difference of about $5 \times 10^{-5} \mathrm{oK}$. Thus very good temperature control would be required; this would be difficult, but not, we believe, impossible, even when we allow for the fact that a powerful laser beam must pass through the liquid.

In this section we have considered the scattering of light by only pure ${ }^{4} \mathrm{He}$ near its $\lambda$-point. Scattering by mixtures of ${ }^{3} \mathrm{He}$ and ${ }^{4} \mathrm{He}$ ought also to be interesting near their $\lambda$-points, and it would be particularly interesting to examine by light scattering the region of the ${ }^{3} \mathrm{He}-{ }^{4} \mathrm{He}$ phase diagram near the intersection of the $\lambda$-line and the phase separation line [42].

\section{References}

[1] LANdAu (L. D.) and Lifshitz (E. M.), The Electrodynamics of Continuous Media, Ch. 14, Pergamon Press, London, 1960.

[2] Landau (L. D.) and Lifshitz (E. M.), Statistical Physics, Ch. 12, Pergamon Press, London, 1958.

[3] Landau (L. D.) and Placzek (G.), Phys. Z. Sov., $1934,5,172$.

[4] Kadanoff (L. P.) and Martin (P. C.), Ann. Phys. $(N . Y), 1963,24,$.

[5] See, for example, FABELINSKII (I. L.), Molecular Scattering of Light, Plenum Press, New York, 1968.

[6] Gornall (W. S.), Stegeman (G. I. A.), StorchefF (B. P.), Stolen (R. H.) and Volterra (V.), Phys. Rev. Letters, 1966, 17, 297.

[7] Mountann (R. D.), J. Res. Natl. Bur. Std., 1966, 70 A, 207.

[8] LANDAu (L. D.) and Lifshitz (E. M.), Fluid Mechanics, pg. 304, Pergamon Press, London, 1959.

[9] Ginsburg (V. L.), Soviet Phys. JETP, 1943, 13, 243.

[10] Bertoletti (M.), de Pasquale (D.) and Sette (D.), Il Nuovo Cim., 1967, 52 B, 560.

[11] Lawson (A. W.) and Meyer (L.), Phys. Rev., 1954, 93, 259.

[12] Grimm (H.) and Dransfeld (K.), Z. Naturforsch., 1967, 22 a, 1629.

[13] Greytak (T. J.), St Peters (R. L.), Benedek (G.), Bull. Am. Phys. Soc.

St Peters (R. L.), Thesis, M. I. T., 1969.

[14] Pike (E. R.), Vaughan (J. M.) and Vinen (W. F.), J. Phys. C., 1970, 3 L, 37.

[15] VAN DEN Berg (G. J.), VAN ItTERbeck (A.), VAN AARDENNE (G. M. V.) and HerfKens (J. H. J.), Physica, 1955, 21, 860 .

[16] Woolf (M. A.), Platzmann (P. M.) and Cohen (M. G.), Phys. Rev. Letters, 1966, 17, 294.
[17] Winterling (G.), Walda (G.) and Heinicke (W.), Phys. Letters, 1968, 26 A, 301.

[18] Abrikosova (I. I.) and Bochkova (O. M.), JETP Letters, 1969, 9, 167.

[19] Heinicke (W.), Winterling (G.) and Dransfeld (K.), Phys. Rev. Letters, 1969, 22, 170.

[20] Imar (J. S.) and Rudnick (I.), Phys. Rev. Letters, 1969, 22, 694.

[21] JACuCCI (G.) and Signorelli (G.), Phys. Letters, $1967,26 \mathrm{~A}, 5$.

[22] Tsintsadze (N. L.), Soviet Phys. JETP, 1969, 28, 950.

[23] See, e. g., Wruks (J.), Liquid and Solid Helium, Oxford University Press, 1967.

[24] Khalatnikov (I. M.), Introduction to the Theory of Superfuidity, Benjamin, New York, 1965.

[25] Gorkov (L. P.) and PitaevskiI (L. P.), Soviet Phys. $J E T P, 1958,6,486$.

[26] Ganguly (G. N.) and Griffin (A.), Can. J. Phys., $1968,46,1895$.

[27] VINEN (W. F.) and VAUGHAN (J. M.), to be published.

[28] Pike (E. R.), Vaughan (J. M.) and Vinen (W. F.), Phys. Letters, 1969, 30 A, 373.

[29] Roberts (T. R.) and Sydoriak (S. G.), Phys. Fluids, $1960,3,895$.

[30] Fairbank (H. A.), Il Nuovo Cim., 1958, suppl. 9 (10), 325 .

McClintock (P. V. E.), Mueller (K. H.), Guyer (R. A.) and Fairbank (H. A.), Proc. 11th Int. Conf. on Low Temp. Phys., St Andrews, 1968, pg. 379.

[31] Halley (J. W.), Phys. Rev., 1969, 181, 338.

[32] Greytak (T. J.) and YAN (J.), Phys. Rev. Letters, $1969,22,987$.

[33] Woods (A. D. B.), Quantum Fluids, éd. D. F. Brewer, North Holland Publ. Co., Amsterdam, 1966, pg. 242. 
[34] Stephen (M. J.), Phys. Rev., 1969, 187, 279.

[35] Dynmin (N. E.), Eselson (B. N.), Rudavskin (E. Ya.) and Serbin (I. A.), Soviet Phys. JETP, 1969, 29, 406.

[36] See, for example, Heller (P.), Rep. Prog. Phys., 1967, 30, 731 .

[37] OrsteIn (L. S.) and Zernike (F.), Proc. Acad. Sci. Amst., 1914, 17, 793.

[38] Kierstead (H. A.), Phys. Rev., 1967, 153, 258.
[39] Barmatz (M.) and Rudnick (I.), Phys. Rev., 1968, 170, 224.

[40] Ferrell (R. A.), Menyhard (N.), Schmidt (H.), Schwabl (F.) and Szépfalusy (P.), Ann. Phys. (N. Y.), 1968, 47, 565 .

[41] Swift (J.) and KADANOFF (L. P.), Ann. Phys. (N. Y.), 1968, 50, 312.

[42] Griffin (A.), Can. J. Phys., 1969, 47, 429. 